\title{
Evaluation of relationship between middle cerebellar peduncle asymmetry and dominant hand by diffusion tensor imaging
}

\author{
G. Polat \\ Department of Radiology, Medical Faculty, Ataturk University, Erzurum, Turkey
}

[Received: 23 October 2018; Accepted: 7 December 2018]

Background: The aim of the study was to evaluate middle cerebellar peduncle (MCP) asymmetry by diffusion tensor imaging (DTI) according to the dominant hand. Materials and methods: Sixty-four volunteers who met the exclusion and inclusion criteria were prospectively evaluated by DTI. Circular voxels of interest were drawn at the right and left MCP levels for all volunteers. The software automatically calculated the median values of fractional anisotropy (FA), mean diffusivity (MD), and number of fibres (NF). The volunteers were categorised as the right-handed $(n=44)$ and left-handed $(n=20)$ groups using the Dellatolas test. The mean values of FA, MD and NF were statistically compared between the two sides and the two groups.

Results: In both groups, the mean value of FA for the right MCP was significantly higher than the left MCP ( $p=0.0016$ and $p=0.0032$ for the right- and left-handed groups, respectively). In both groups, no significant difference was observed between the right and left MCPs in terms of MD and NF ( $p=0.75$, $p=0.69$ and $p=0.96, p=0.46$, respectively). There was also no statistically significant difference between the groups for the mean values of FA, MD and NF of each MCP $(p=0.74, p=0.26$ and $p=0.26$, respectively for the right MCP and $p=0.78, p=0.37$ and $p=0.54$, respectively for the left $M(P)$.

Conclusions: There is an asymmetry between the mean FA values of the right and left MCP. However, this asymmetry is independent of the dominant hand. (Folia Morphol 2019; 78, 3: 481-486)

Key words: middle cerebellar peduncle, diffusion tensor imaging, fractional anisotropy, dominant hand

\section{INTRODUCTION}

The cerebellum plays a key role in many functions; e.g., supporting cerebral functions in coordination, cognitive and motor skills, and mediating coordination by establishing connections with the cerebral cortex, limbic system, and thalamus [19]. Middle cerebellar peduncle (MCP) is the major input pathway of the cerebellum [14, 19]. Morphological changes in the cerebellum and pathways are observed in movement disorders, such as Parkinson's disease [21]. Studies using diffusion tensor imaging (DTI) have shown fraction anisotropy (FA) changes in these pathways in patients with movement disorders [15]. Therefore, DTI is an important modality for the evaluation of these pathways. It is a powerful technique, especially for studying the microstructure of white matter [19]. DTI examines

Address for correspondence: Dr. G. Polat, Department of Radiology, Faculty of Medicine, Ataturk University, 25040 Erzurum, Turkey, tel: +90 442344 6920, fax: +90 442 2361014, e-mail: dr.g.polat@gmail.com 
the molecular diffusion of water and provides the FA value, which is a quantitative diffusion parameter associated with axonal packing and myelination [19]. Since this axonal packing and myelination is responsible for the neural connection, it contributes greatly to the understanding of the pathways between the brain regions of DTI measurements [19].

In consecutive hand movements, the corticospinal and MCP tracts work in coordination $[12,15,16]$. It has been reported that there are functional and structural differences in the cerebral hemispheres of the right- and left-handed patients $[4,5,10,11,16]$. In corticospinal tract evaluation using DTI, asymmetry was observed to be associated with the dominant hand $[12,16]$. Similarly, van der Zwaag et al. [18] found that lateralised hand motor movement has a greater impact on the cerebellar lobules 5 and 8 more. This suggests that the dominant hand can affect cerebellar pathways providing motor coordination. However, in the literature, there is no study that used DTI to evaluate the cerebellar pathways coordinating sequential motor activity in relation to the dominant hand. Therefore, in this study, we aimed to evaluate MCP asymmetry by DTI according to the dominant hand.

\section{Study design}

This was a prospective cohort DTI study. All participants provided written informed consent and the study protocol was approved by the institutional Medical Ethics Committee. Eighty-two healthy volunteers aged $15-65$ years were enrolled in the study. The individuals were excluded if they reported a neurological disorder $(n=3)$ (stroke, haemorrhage and transient ischaemic attack) or a medical disorder $(n=6)$ (diabetes mellitus, hypertension and other vascular diseases), history of mental retardation $(n=1)$, drug abuse or dependence $(n=1)$, psychiatric illness $(n=2)$, and magnetic resonance imaging (MRI) contraindications $(n=5)$. The remaining 64 volunteers were included in the study. The mean age of the patients was $38.5 \pm 17.2$ years. Thirty-one volunteers were male and 33 were female. Dominant hand was determined using a test created by Dellatolas et al. [3]. According to the results, 44 volunteers were categorised as right-handed and 20 as left-handed.

\section{Diffusion tensor imaging}

The volunteers were placed on the magnetic resonance (MR) table in a supine position. All MRI studies were acquired using a 1.5 T MR imaging scanner (Magnetom area; Siemens Healthcare, Berlin, Germany). A three-dimensional T1-weighted magnetisation-prepared rapid gradient echo (MP-RAGE) sequence was used for conventional MRI and a sensitivity-encoded single-shot echo-planar imaging (SENSEEPI) sequence was used for DTI. The sequence parameters for MP-RAGE were as follows: voxel size $1 \times 1 \times 1 \mathrm{~mm}$, slice thickness $1.15 \mathrm{~mm}$, field-of-view (FOV) $256 \times 90$, repetition time/echo time $(T R / T E)=1420 / 2.98 \mathrm{~ms}$, flip angle 9 , and matrix $186 \times 256$. The SENSEEPI protocol parameters were: TR/TE $=3700 / 95 \mathrm{~ms}$, FOV $240 \times 100$, voxel size $1.9 \times 1.9 \times 4 \mathrm{~mm}$, and slice thickness $4 \mathrm{~mm}$. We used 20 directional diffusion-weighted scans with a b-value of $1000 \mathrm{~s} / \mathrm{mm}^{2}$.

\section{Evaluation of DTI data}

The resulting MRI were assessed on a workstation (Syngo via, Siemens Medical Imaging, Erlangen, Germany). Our offline post-processing system uses deterministic tractography. A radiologist (G.P.) with 7 years of experience evaluated the DTI data. Circular voxels of interest $(1 \mathrm{~cm}$ radius) were placed on bilateral MCPs to obtain and analyse the DTI values (Fig. 1). The software automatically calculated the median values of FA, mean diffusivity (MD), and number of fibres (NF) of bilateral MCPs. The same radiologist repeated the measurements for each volunteer on 3 different days (minimum interval between each measurement was 1 week). The data were evaluated by calculating the average values.

\section{Statistical analysis}

All statistical analyses were performed using MedCalc software (version 12.2.1.0, Mariakerke, Belgium). Distribution of variables assessed with D'Agostino Pearson test. Comparison between two groups with normal distribution performed with independent $t$ test. Nominal categorical variables were compared using $\chi^{2}$ test. In both right-handed and left-handed groups, the statistical significance of the differences between the mean values of FA, MD and NF were assessed by the Mann-Whitney $U$ test. For the right-MCP and left-MCP groups, this evaluation was undertaken using the Wilcoxon test.

\section{RESULTS}

The percentage of men $43.2 \%(n=19)$ in the right-handed group and $60 \%$ in the left-handed group $(n=12)(p=0.96)$. The mean ages were 

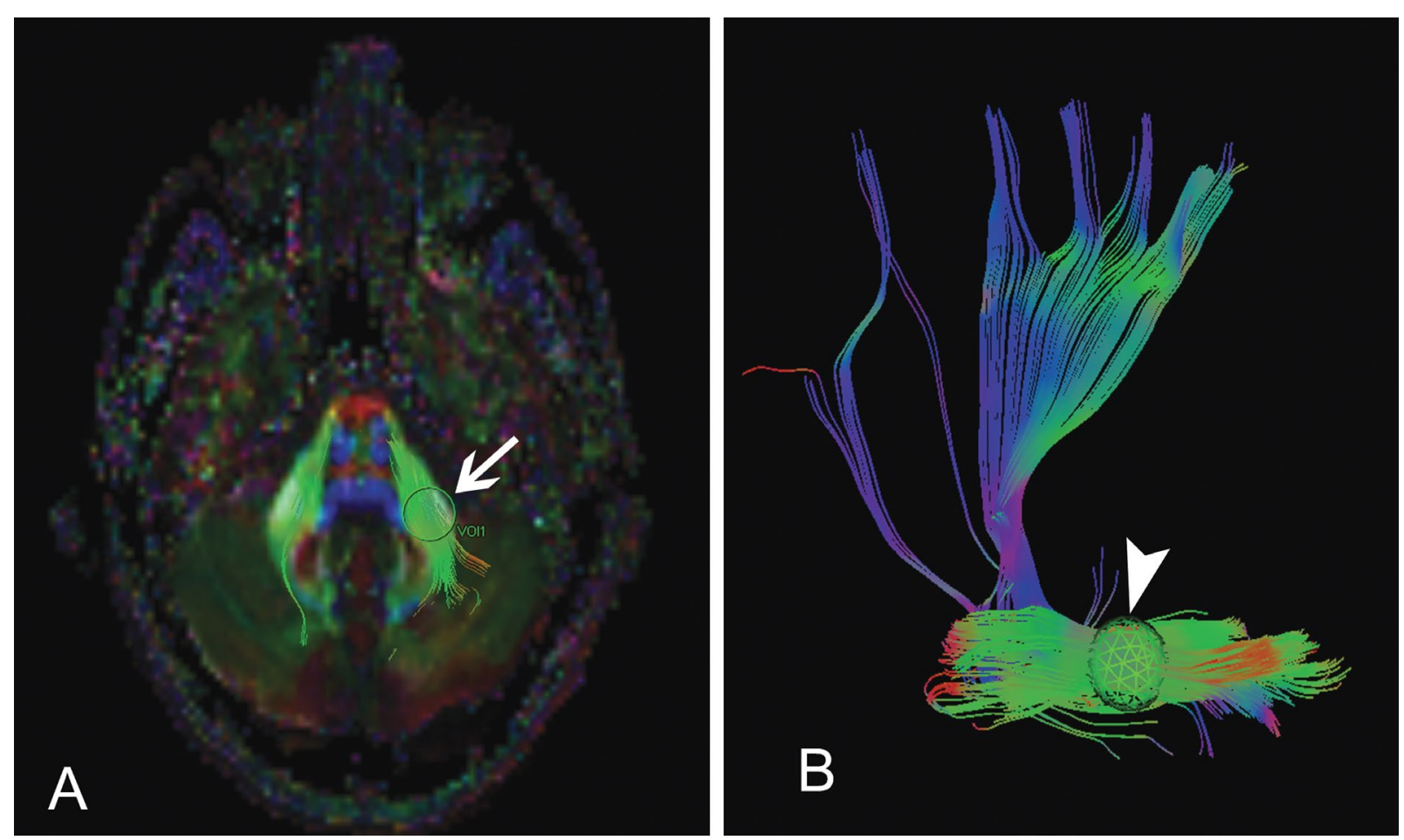

Figure 1. Diffusion tensor imaging shows right middle cerebellar peduncle (white arrow) in whole tracts $(\mathbf{A})$. In addition, three-dimensional image shows tracts of middle cerebellar peduncle (arrowhead) (B); VOI — voxels of interest.

Table 1. Statistical results of right-handed and left-handed groups

\begin{tabular}{|c|c|c|c|c|c|c|c|}
\hline & \multicolumn{3}{|c|}{ Right-handers } & \multicolumn{3}{|c|}{ Left-handers } & Intergroup comparison (p) \\
\hline Age & \multicolumn{3}{|c|}{$36.7 \pm 17.8$} & \multicolumn{3}{|c|}{$42.5 \pm 17.05$} & 0.10 \\
\hline Sex (male ratio) & \multicolumn{3}{|c|}{$19(43.2 \%)$} & \multicolumn{3}{|c|}{$12(60 \%)$} & 0.96 \\
\hline & Right MCP & Left MCP & $\mathbf{P}$ & Right MCP & Left MCP & $\mathbf{P}$ & Right and left \\
\hline Fractional anisotropy & 0.49 & 0.47 & 0.0016 & 0.48 & 0.47 & 0.0032 & 0.74 and 0.78 \\
\hline Mean diffusivity & 0.0008 & 0.0008 & 0.75 & 0.0008 & 0.0008 & 0.69 & 0.26 and 0.26 \\
\hline Number of fibres & 387 & 376 & 0.96 & 378 & 365 & 0.46 & 0.37 and 0.54 \\
\hline
\end{tabular}

MCP — middle cerebellar peduncle

$36.7 \pm 17.8$ years and $42.5 \pm 17.05$ years for the right- and left-handed groups, respectively $(p=0.10)$. The data of both groups are shown in Table 1. In both groups, the value of FA in the right MCP was significantly higher compared to the left MCP ( $p=0.0016$ and $p=0.0032$, respectively) (Figs. 2, 3). However, neither group had any significant difference between the right and left MCPs in terms of MD and NF (Table 1). There was also no significant difference between the right- and left-handed volunteers concerning FA, MD, and NF (Table 1).

\section{DISCUSSION}

This study showed that there was no significant difference between the right- and left-handed indi- viduals in terms of FA, MD and NFin the MCP motor activity pathway. A few studies have shown functional hemispheric asymmetry between right- and left-handed people $[10,11]$. These studies have demonstrated a relationship between handedness and anatomical or functional asymmetry of the brain. Recently, asymmetry between grey and white matter of the encephalic volume was revealed through cross-sectional imaging $[6,13]$. In the evaluation of the cerebral hemisphere according to the dominant hand, the differences in FA and MD secondary to asymmetry were revealed on DTI $[2,8,16,20]$. However, in our study, we found no statistically significant difference between the right- and left-handed groups for the investigated parameters. Seizeur et al. [16] showed that the FA 

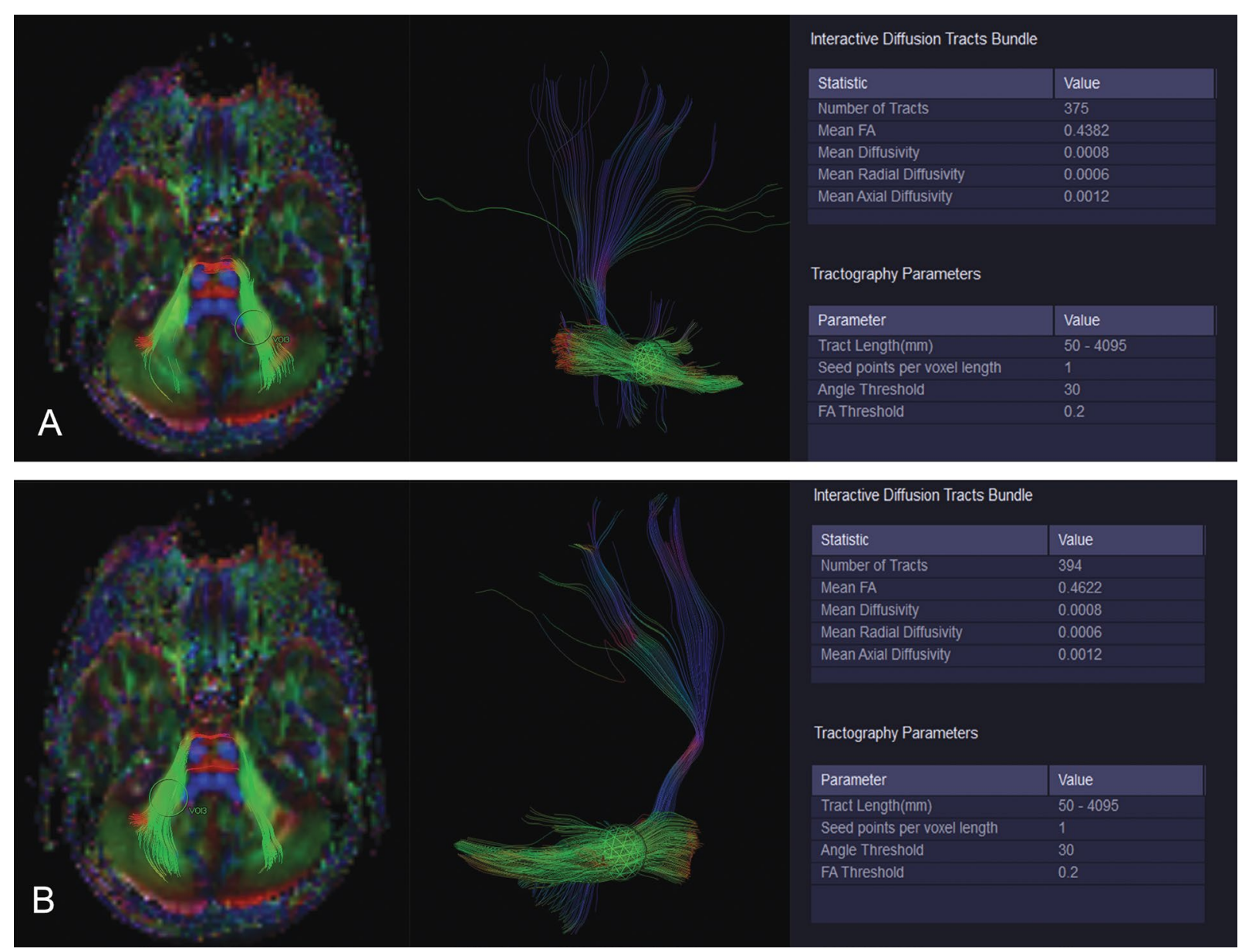

Figure 2. Diffusion tensor imaging shows evaluation of left (A) and right (B) middle cerebellar peduncle by voxels of interest in the right-handed volunteer. Right middle cerebellar peduncle fractional anisotropy (FA) is higher than left.

values (right and left corticospinal tract) of the right handers were significantly higher than those of the left handers at the levels of the internal capsule and diencephalon-mesencephalon junction. However, the authors found no significant difference between the two groups for the FA values in the left corticospinal tract at the level of the corona radiate [16]. The current study showed that the FA differences between the dominant and non-dominant hands occurred due to anatomical location. The lack of a lateralisation finding due to anatomic localisation in the corticospinal tracts that conduct and direct motor activation can explain the absence of the same finding in the coordination tracts. Many studies reported that asymmetry due to dominant hand affects anatomic volumes $[1,7,17]$. On the other hand, Kavaklioglu et al. [9] revealed no significant relationship between dominant hand and cerebellar anatomic asymmetry. Similar to the other anatomical studies in the literature, we also did not determine any effect of the dominant hand on the asymmetry of the cerebellar pathways.

In the current study, it was observed that the FA values in the right MCP were significantly higher than those in the left MCP, regardless of the dominant hand. Re et al. [14] showed significant differences between the right and left MCP motor tracts in terms of FA and NF. However, no significant difference was observed in MCP cognitive tracts [14]. Although their study did not make an evaluation of the dominant hand, it was important in terms of demonstrating the possibility of an asymmetry at the MCP level. In the current study, no significant asymmetry was observed for MD and NF. Re et al. [14] reported no significant difference between the MCP cognitive tracts in terms of NF, but a significant difference was noted for the MCP motor tracts. In the current study, we evaluated the MCP motor and cognitive tracts together considering that motor coordination is the result of the harmony of a system that connects cerebral and cerebellar cortical areas as 

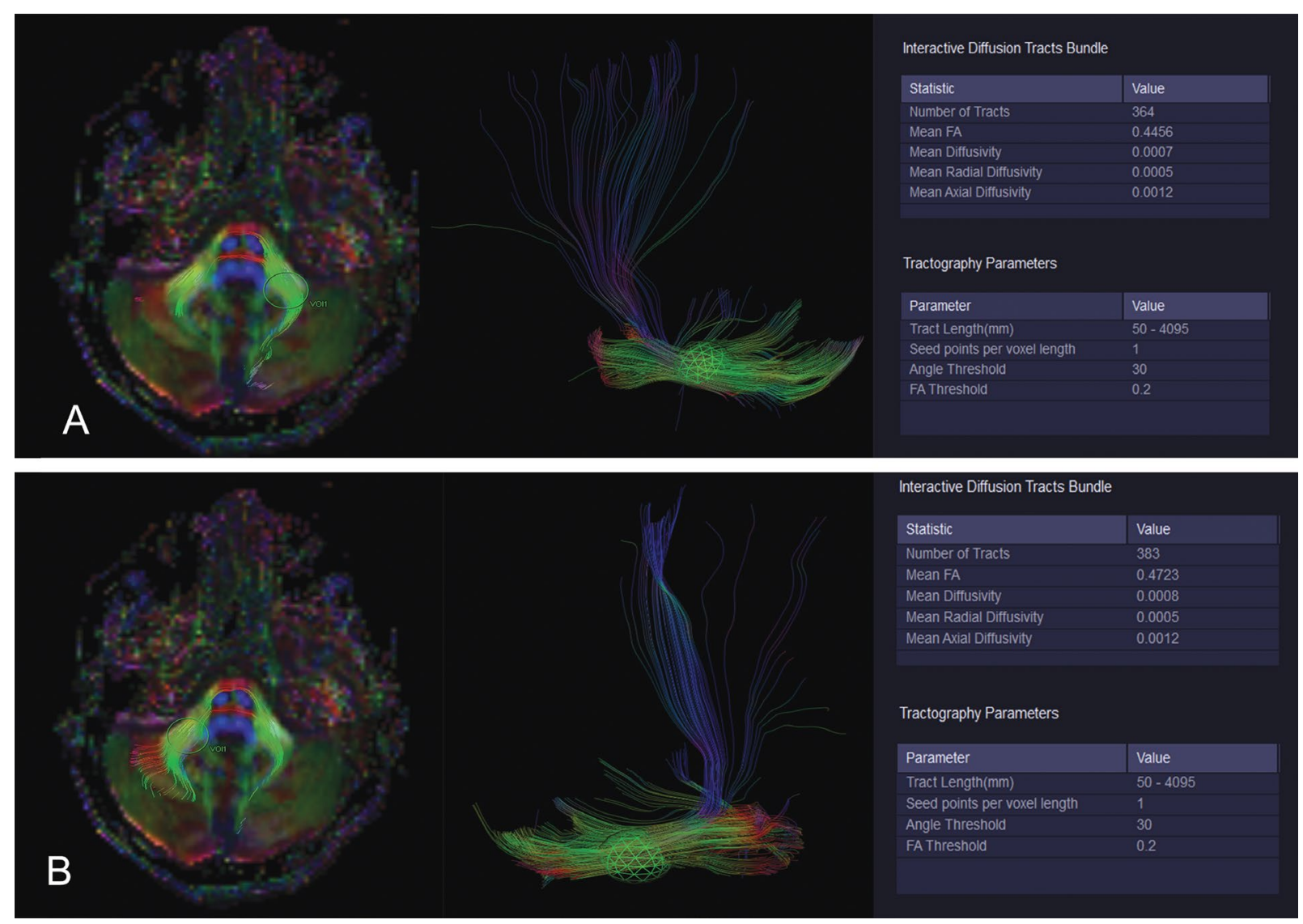

Figure 3. Diffusion tensor imaging shows evaluation of left (A) and right (B) middle cerebellar peduncle by voxels of interest in the left-handed volunteer. Right middle cerebellar peduncle fractional anisotropy (FA) is higher than left.

a circle $[14,19]$. Since we evaluated these pathways together, we may not have observed any asymmetry of the subgroup pathways in terms of MD and NF.

This study had certain limitations. First, we had a relatively low number of volunteers. In addition, we did not make our assessments according to the subgroup pathways. Therefore, we were not able to evaluate the association between asymmetries in the MCP subgroup pathways and dominant hand. Third, we have used standard region of interest for tracking the MCP in all cases. This could cause covering of other fibre groups that are unrelated with MCP, thus lowering the reliability of our results. However, we think that these minor add-ons could not change our significance of comparisons.

\section{CONCLUSIONS}

In conclusion, the asymmetry of FA was observed in cerebellar pathways, which play an effective role in motor coordination. However, the asymmetry of these tracts was independent of dominant hand according to DTI results.

\section{Acknowledgements}

We would like to thank insan Yüce, Ahmet Yalçın and Recep Sade for assistance with data acquisition and analysis.

\section{REFERENCES}

1. Amunts K, Schlaug G, Schleicher A, et al. Asymmetry in the human motor cortex and handedness. Neuroimage. 1996; 4(3 Pt 1): 216-222, doi: 10.1006/nimg.1996.0073, indexed in Pubmed: 9345512.

2. Büchel C, Raedler T, Sommer M, et al. White matter asymmetry in the human brain: a diffusion tensor MRI study. Cereb Cortex. 2004; 14(9): 945-951, doi: 10.1093/cercor/ bhh055, indexed in Pubmed: 15115737.

3. Dellatolas GD, Jallon $P$, Poncet $M$, et al. Mesure de la préférence manuelle par autoquestionnaire dans la population française adulte. Psychologie aplliquee. 1988; 38: 117-136.

4. Galaburda AM, Sanides F, Geschwind N. Human brain. Cytoarchitectonic left-right asymmetries in the temporal speech region. Arch Neurol. 1978; 35(12): 812-817, indexed in Pubmed: 718483.

5. Geschwind N. Cerebral dominance and anatomic asymmetry. N Engl J Med. 1972; 287(4): 194-195, doi: 10.1056/ NEJM197207272870416, indexed in Pubmed: 5033533.

6. Good CD, Johnsrude I, Ashburner J, et al. Cerebral asymmetry and the effects of sex and handedness on brain 
structure: a voxel-based morphometric analysis of 465 normal adult human brains. Neuroimage. 2001; 14(3): 685-700, doi: 10.1006/nimg.2001.0857, indexed in Pubmed: 11506541.

7. Guadalupe T, Willems RM, Zwiers MP, et al. Differences in cerebral cortical anatomy of left- and right-handers. Front Psychol. 2014; 5: 261, doi: 10.3389/fpsyg.2014.00261, indexed in Pubmed: 24734025.

8. Hammond G. Correlates of human handedness in primary motor cortex: a review and hypothesis. Neurosci Biobehav Rev. 2002; 26(3): 285-292, indexed in Pubmed: 12034131.

9. Kavaklioglu T, Guadalupe T, Zwiers M, et al. Structural asymmetries of the human cerebellum in relation to cerebral cortical asymmetries and handedness. Brain Struct Funct. 2017; 222(4): 1611-1623, doi: 10.1007/s00429016-1295-9, indexed in Pubmed: 27566607.

10. Kertesz A, Geschwind N. Patterns of pyramidal decussation and their relationship to handedness. Arch Neurol. 1971; 24(4): 326-332, indexed in Pubmed: 5548452.

11. Kertesz A, Polk $M$, Black SE, et al. Anatomical asymmetries and functional laterality. Brain. 1992; 115 (Pt 2): 589-605, doi: 10.1093/brain/115.2.589, indexed in Pubmed: 1606483 .

12. Lee DH, Lee DW, Han BS. Symmetrical location characteristics of corticospinal tract associated with hand movement in the human brain: a probabilistic diffusion tensor tractography. Medicine (Baltimore). 2016; 95(15): e3317, doi: 10.1097/MD.0000000000003317, indexed in Pubmed: 27082576.

13. Pujol J, López-Sala A, Deus J, et al. The lateral asymmetry of the human brain studied by volumetric magnetic resonance imaging. Neuroimage. 2002; 17(2): 670-679, indexed in Pubmed: 12377142.

14. Re TJ, Levman J, Lim AR, et al. High-angular resolution diffusion imaging tractography of cerebellar pathways from newborns to young adults. Brain Behav. 2017; 7(1): e00589, doi: 10.1002/brb3.589, indexed in Pubmed: 28127511.

15. Reimão S, Morgado C, Neto L, et al. Diffusion Tensor Imaging in Movement Disorders: Review of Major Patterns and Correlation with Normal Brainstem/cerebellar White Matter. Neuroradiol J. 2011; 24(2): 177-186, doi: 10.117 7/197140091102400203, indexed in Pubmed: 24059605.

16. Seizeur R, Magro E, Prima S, et al. Corticospinal tract asymmetry and handedness in right- and left-handers by diffusion tensor tractography. Surg Radiol Anat. 2014; 36(2): 111-124, doi: 10.1007/s00276-013-1156-7, indexed in Pubmed: 23807198.

17. Toga AW, Thompson PM. Mapping brain asymmetry. Nat Rev Neurosci. 2003; 4(1): 37-48, doi: 10.1038/nrn1009, indexed in Pubmed: 12511860.

18. van der Zwaag W, Kusters R, Magill A, et al. Digit somatotopy in the human cerebellum: a $7 \mathrm{~T} \mathrm{FMRI}$ study. Neuroimage. 2013; 67: 354-362, doi: 10.1016/j.neuroimage.2012.11.041, indexed in Pubmed: 23238433.

19. Wang S, Fan GG, Xu Ke, et al. Altered microstructural connectivity of the superior and middle cerebellar peduncles are related to motor dysfunction in children with diffuse periventricular leucomalacia born preterm: a DTI tractography study. Eur J Radiol. 2014; 83(6): 997-1004, doi: 10.1016/j.ejrad.2014.03.010, indexed in Pubmed: 24703518.

20. Westerhausen R, Huster RJ, Kreuder F, et al. Corticospinal tract asymmetries at the level of the internal capsule: is there an association with handedness? Neuroimage. 2007; 37(2): 379-386, doi: 10.1016/j.neuroimage.2007.05.047, indexed in Pubmed: 17601751.

21. Wu T, Hallett M. The cerebellum in Parkinson's disease. Brain. 2013; 136(Pt 3): 696-709, doi: 10.1093/brain/ aws360, indexed in Pubmed: 23404337. 